\title{
Impact of Dementia on Mindful Attention: a Cross-Sectional Comparison of People with Dementia and Those Without
}

\author{
Catherine Bousfield ${ }^{1} \cdot$ Joshua Stott $^{2}$ (D)
}

Published online: 26 June 2018

(C) The Author(s) 2018

\begin{abstract}
Mindfulness-based interventions have been suggested as ways of improving mood and cognition in people with dementia. Existing findings suggest possible benefit from a mindfulness-based group intervention. However, it is unclear whether any structured group activity (rather than mindfulness practice per se) would produce the same benefits, particularly since dementia may impact mindful attention ability. Consequently, we investigated the potential impact of having dementia on mindful attention. We compared the performance of 34 people with dementia recruited from memory services with 55 communityrecruited older people on measures of mindful attention, cognitive flexibility, and cognition, as well as putative nuisance variates of depression, anxiety and premorbid intellectual ability. The groups differed significantly on a range of demographic characteristics and some neuropsychological and mood measures. However, neither the primary prediction (that there would be a large effect size difference between groups, with people with dementia performing significantly more poorly on a measure of mindful attention to the breath), nor the secondary prediction (that performance on this measure would positively correlate with measures of executive function and overall cognition) was supported. We concluded that a diagnosis of dementia may not have a large effect on mindful attention, with consequent implications for future research.
\end{abstract}

Keywords Mindfulness $\cdot$ Meditation $\cdot$ Dementia $\cdot$ Attention $\cdot$ Cognition $\cdot$ Cognitive flexibility

\section{Introduction}

Dementia is an umbrella term for a number of progressive and chronic syndromes, with underlying neurological causes and varying patterns of impairment in cognitive function, as well as emotional and behavioural changes (World Health Organization 2016). It is estimated that 46.8 million people worldwide are living with the condition (Prince et al. 2015). The economic cost of the condition is high (estimated at $£ 26.3$ billion a year in the UK alone (Prince et al. 2014)), as is the biopsychosocial impact on people living with dementia and those who care for

Joshua Stott

j.stott@ucl.ac.uk

1 St George's University Hospitals NHS Foundation Trust, London, UK

2 UCL Research Department of Clinical, Educational \& Health Psychology, University College London, 1-19 Torrington Place, London WC1E 6BT, UK them. In addition to cognitive impairment, people with dementia experience depression at far higher rates than the non-dementia population (Winblad et al. 2004, cited in Enache et al. 2011; Kales et al. 2005), with damaging effects (González-Salvador et al. 1999; Kunik et al. 2003; Orgeta et al. 2015; Rapp et al. 2011; Shin et al. 2005; Suh and Yeon 2005).

Given the evidence that mindfulness-based interventions can reduce current depressive symptoms, reduce risk of future depression and enhance cognition in those without dementia (Chiesa et al. 2011; Piet and Hougaard 2011; Strauss et al. 2014), there has been increasing interest in the application of mindfulness-based interventions to those with dementia. The most recent and rigorously conducted trial in people already diagnosed with dementia suggested that an adapted mindfulness-based stress reduction intervention can improve self-reported quality of life in those living with dementia in care homes (Churcher Clarke et al. 2017). However, the mechanism by which this improvement was obtained was unclear, as there was no improvement in mindfulness skills over the course of the intervention. We know that stimulating structured group activity may produce similar results to those 
found in Churcher Clarke et al. (Spector et al. 2003), thus it may be that any such activity (rather than mindfulness practice in particular) would produce the same benefits.

It is also possible that the cognitive deficits present in dementia may preclude or at least affect the ability to practice mindfulness, rather than to engage in structured activity per se. The issue of whether such deficits do affect mindfulness ability has not previously been investigated. It is important to understand both theoretically and practically, as mindfulness-based stress reduction is a highly specialised intervention requiring expert facilitation with consequent cost and resource implications, whereas other structured group interventions are not (mindfulnessteachersuk.org.uk 2011; Spector et al. 2003). In particular, the ability to attend (and to self-regulate attention) has been identified as one of the core components of mindfulness practice and the related construct of cognitive flexibility is empirically associated with mindfulness ability (Bishop et al. 2006; Moore and Malinowski 2009). Cognitive flexibility and attentional regulation are also affected early in the disease process of the two most common forms of dementia, Alzheimer's disease and vascular dementia (World Health Organization 2016).

Consequently, in the current study, we aimed to compare ability on a mindful attention task in a group of people with dementia and in an older adult comparison group. To enhance ecological validity, we did this using an experience sampling measure (Napa Scollon et al. 2009), the Meditation Breath Attention Score (MBAS) (Frewen et al. 2008). The MBAS task has been used in a dementia sample previously (Churcher Clarke et al. 2017) and was selected in order to minimise the memory load which exacerbates issues with retrospective selfreport of skills in this population, while still replicating core elements of the common meditation practice of mindfulness of breathing. Our primary prediction was that having dementia would have a large effect size impact on mindful attentional ability. Given that we expected this effect to be due to cognitive deficits we made a secondary prediction that, within the dementia sample, levels of overall cognition and cognitive flexibility would be correlated with mindful attention skill levels, and that if these correlations were present, it would be expected that overall cognition and cognitive flexibility predicted mindfulness performance over and above mood and premorbid IQ.

\section{Method}

\section{Participants}

This study reports on a subsample of a cohort study examining therapy abilities in those with dementia. The first study using the cohort has been published (Stott et al. 2017a). However, as this study was on a subsample with different participant numbers and measures, inclusion/exclusion criteria and recruitment procedures are reported below.

\section{Sample Size Calculation}

Sample size was calculated using G*Power (Kiel 2007) for the primary hypothesis that there would be a significant difference in MBAS between people with dementia and the older adult comparison group. Previous work suggested a large effect size (Hebblethwaite et al. 2011). However, given the differences in that study's population, we powered our study to detect an effect size between medium and large (Cohen's $d=0.6$ ) (Cohen 1992) using an ANOVA. The required sample at a 2 (older adults): 1 (dementia) ratio, power $=0.8$, alpha $=0.05$ was 54 older adults and 27 people with dementia.

The dementia sample comprised a consecutive referrals sample of 34 participants diagnosed with mild dementia (last MMSE score $\geq 24$ or ACE $>70$ equivalent on other cognitive screen (Law et al. 2013). Diagnosis had been reached via psychiatrist-led MDT discussion in memory clinics, supported by interpretation from experienced clinical psychologists specialising in clinical neuropsychology. The main diagnosis in the dementia sample was Alzheimer's disease, with one participant diagnosed with dementia in Parkinson's disease, and three with dementia type unspecified. The comparison group comprised a convenience sample of 55 people aged over 65 who by self-report were healthy with no subjective memory problems or a diagnosis of dementia. Where comparison group participants scored below the threshold score of 82 on the ACE-III (where dementia is detected with $100 \%$ specificity, (Hsieh et al. 2013)), their data were excluded from analysis. The sample was recruited by advertisement in community groups and snowball sampling. Participants were primarily tested in an academic setting.

\section{Inclusion Criteria}

Participants were required to be fluent in English. It was also a prerequisite that they had the capacity to consent to participation.

\section{Exclusion Criteria}

Self-reported previous experience of mindfulness meditation practice; recorded or observed as displaying current significant mood/anxiety disorders, psychotic symptoms or substance misuse problems; premorbid learning disability; sensory or physical disabilities or impairments which would impede engagement with testing. As this study drew on a dataset which will also be used to examine the relationship of neuropsychological functioning to Cognitive Behavioural Therapy (CBT), participants reporting experience of CBT were also excluded. 


\section{Procedure}

Demographic information was obtained at the beginning of testing from all participants. For the dementia group, this information was verified via electronic healthcare records. The order of presentation for mindfulness and neuropsychological measures was counterbalanced, and the ordering of neuropsychological measures randomised, to minimise order effects. For the mindful attention measure, a recorded version of the task was used to ensure consistency of delivery across participants. Whether participants were asked to raise their left or right hand to signify attending/non-attending to the breath was counterbalanced across the sample to reduce the potential influence of dominant hand on response choice.

Test batteries were administered in clinics or participants' homes by trainee clinical psychologists and psychology graduates trained in administration and scoring of the measures. Supervision was provided by a clinical psychologist with a postgraduate diploma in clinical neuropsychology.

All testing was conducted in compliance with ethical guidelines on human experimentation (World Medical Association 2013). Ethical approval was given by NRES Committee London-City Road \& Hampstead (REC Reference 14/LO/0554). Data were collected between August 2015 and February 2016.

\section{Measures}

\section{Meditation Breath Attention Score Task}

The meditation breath attention score (MBAS) task (Frewen et al. 2008) is assessed via a 15-min exercise (following a 10min practice period) in which participants are instructed to keep their attention on their breath, noticing without judgement if their attention wanders, and then returning their attention to the breath. A bell is rung at 3-min intervals, with participants instructed to raise one hand if their attention is on the breath, and the other hand if it is elsewhere. The response is recorded by the tester and the exercise then continues. The number of times each participant indicates that they were focused on the breath is recorded (with a maximum possible score of five). Studies have demonstrated convergent validity (Frewen et al. 2008; Liu et al. 2013), construct validity (Frewen et al. 2011; Lai et al. 2015) and test-retest reliability (Frewen et al. 2014).

Use of a simplified version of the MBAS task (requiring shorter periods of concentration, and offering additional attentional cues), adapted to reduce task demands for a more severely impaired nursing home dementia sample (Churcher Clarke et al. 2017) was considered for the current study. The lead author informally piloted the simplified task as a pre-post measure for a psychoeducation/wellbeing group comprising six people with mild dementia. The mean MMSE at group entry was 23.5 , compared to a mean MMSE of 15.85 in the nursing home sample.

It was found in the pilot that group members scored at or near ceiling on the measure (with a mean score of 4/5), with very little variability. Qualitative feedback indicated that scores were congruent with intended responses, and the group felt that the task was simple. Based on these observations, it was judged that the simplified measure would be unlikely to have utility for identifying difference within the current study's dementia group (and the same would have applied for the non-dementia comparison group). Therefore, it was decided to use the standard version of the MBAS task.

\section{Trail Making Test}

Cognitive flexibility was measured using the trail making test (TMT), a two-part measure, frequently used with and acceptable to people with dementia, with excellent inter-rater reliability (Bowie and Harvey 2006). Participants are asked to 'join the dots' on a series of targets. In trail A, this is a series of sequential numbers; in trail B, a series of alternating sequential numbers and letters. Trail B has support as a measure of executive function (specifically, cognitive flexibility), and has been shown to significantly discriminate subjects with and without dementia (Heun et al. 1998; Kortte et al. 2002). Participants' levels of cognitive flexibility is understood to be best represented by using a score derived from combining trails A and B scores, to reduce the impact of factors such as reduced motor and visual scanning speed on the trail B setswitching task. Sanchez-Cubillo et al. (2009) suggests that the difference score (trail B score minus trail A score) is the most accurate reflection of set-switching ability.

\section{Addenbrooke's Cognitive Examination-III}

The Addenbrooke's Cognitive Examination-III (ACE-III) was used to assess overall cognition. It is a widely used and acceptable measure for people with dementia and is frequently used as a screening tool within memory services. It has been validated against its predecessor the ACE-R and other standardised tests of neuropsychological functioning in early dementia (Hsieh et al. 2013), and has been shown to have high internal reliability (Cronbach's $\alpha=0.88$ (Velayudhan et al. 2014)).

\section{Hospital Anxiety and Depression Scale}

Anxiety and depression were seen as potential nuisance variates in data analysis, since both can influence cognition (Austin 2001; Derakshan and Eysenck 2009), and depression may directly impact performance on mindfulness and cognitive measures via reduced motivation and effort (Lezak et al. 2012). Therefore, the Hospital Anxiety and Depression Scale 
(HADS) (Snaith and Zigmond 1983) was administered to all participants to identify clinically relevant levels of anxiety or depression. The HADS is an easy-to-administer, wellaccepted questionnaire measure consisting of 14 self-report items equally contributing to two seven-item subscales assessing depression and anxiety. As it does not assess somatic symptoms of depression there is a reduced likelihood of false positives in screening populations with poorer physical health. Higher scores correspond to higher disease severity (Herrero et al. 2003; Johnston et al. 2000). The measure has acceptable internal consistency, is feasible for use with dementia patients and has demonstrated good validity in assessing symptom severity and caseness of anxiety and depression across populations (Bjelland et al. 2002; Samaras et al. 2013; Stott et al. 2017b).

\section{Test of Premorbid Functioning}

Evidence suggests that for people with intellectual disability, IQ level is related to poorer metacognitive ability (Dagnan and Mellor 2009; Joyce et al. 2006). It might therefore be suspected that in people with dementia, lower premorbid IQ would affect ability to perform a mindfulness task, beyond any impact of specific dementia-related deficits. Estimated premorbid IQ was therefore assessed as a potential nuisance variate in the data. Estimated premorbid scores were derived from the score on the Test of Premorbid Functioning (TOPF) (Wechsler 2011), along with demographic information (gender, years of education). The TOPF requires the reading and pronunciation (but not comprehension) of words with irregular grapheme-to-phoneme translation, which is relatively well preserved in early dementia (Holdnack et al. 2013). The TOPF was validated as part of the wider WAIS-IV/WMS-IV UK validation process, which included a group study of people with probable Alzheimer's disease. Results were in line with the prediction that TOPF-predicted IQ and memory would be higher than the obtained WMS and WAIS scores, suggesting it has utility as a measure of premorbid IQ in this population.

\section{Data Analyses}

Data were analysed using SPSS version 22. A cross-sectional between groups design was used, with MBAS performance of the sample of people with dementia and the healthy older adult group compared using a Mann-Whitney $U$ test, since assumptions for parametric testing were not met. A cross-sectional correlational design was used to evaluate associations between the mindful attention measure and neuropsychological measures. Pearson correlations were used where assumptions were met with non-parametric equivalents applied in other cases. Analyses of continuously varying demographic differences between the groups for the primary prediction were made using independent groups $t$ tests, with chi-square/
Fisher's exact tests used to compare the groups on categorical variables.

\section{Results}

Demographic characteristics of the samples are presented in Table 1. Those with dementia were older and less educated than those in the comparison group $(p<0.001)$. Other demographic characteristics did not differ between groups.

Data for neuropsychological and mood variables are shown in Table 2. For the dementia group, there was a small amount of missing data on some variables (one participant's response in each case). Given the low level of missing data, which were missing completely at random (Little's MCAR $p>0.05$ ), data were deleted listwise (Graham 2009). For the MBAS task, there was a greater percentage of missing/ambiguous responses. Twenty-four percent (8/34) of participants had appeared to respond impulsively (raising their hand before the instructions were given), incongruently (raising the hand indicating attention to the breath when the bell rang, but commenting later that they had not been attending), ambiguously (half raising a hand then putting it down), or not at all. It was felt that listwise deletion, excluding these eight cases from all analyses involving the MBAT, was likely to have too great an effect on the power of the study and would further increase the risk of Type II error. Therefore for this variable only, missing/ambiguous responses were imputed. While several methods for doing this were considered (Gelman and Hill, 2006; Higgins and Green, 2011), the final decision was to impute assumed scores of ' 0 ', equivalent to participants having indicated they were not attending to the breath at the bell.

The analysis for the primary prediction (that there would be a large effect size difference on MBAS performance between groups, with better performance in healthy older adults than in those with dementia) was then run twice: once with imputed data included $(N=34)$, and once with listwise deletion of participants who had given missing/ambiguous MBAS task responses $(N=26)$. MBAS data for the healthy comparison group were complete $(N=55)$.

Full results for the primary prediction are given in Table 3 . No statistically significant difference was found between the groups $(p=0.38)$, and this difference remained insignificant whether or not participants with imputed MBAS data were included in the analysis $(p=0.79)$. Therefore the null hypothesis (that people with dementia and those without would not differ on MBAS performance and that the groups had come from a population with the same median score) could not be rejected. The effect sizes of both calculations $(r=0.09, r=$ 0.03 ) were below the accepted conventional cut-off for a 'small' effect size of 0.1 (Cohen 1992).

The secondary prediction was that MBAS performance would positively correlate with measures of executive 
Table 1 Demographic characteristics and results of between group analyses for people with dementia (PWD) and older adult (OA) participants

\begin{tabular}{|c|c|c|c|c|}
\hline & & \multicolumn{2}{|l|}{ Group } & \\
\hline & & $\operatorname{PWD}(N=34)$ & $\mathrm{OA}(N=55)$ & \\
\hline \multicolumn{2}{|l|}{ Variable } & M (SD) & $\mathrm{M}(\mathrm{SD})$ & Test statistic \\
\hline \multicolumn{2}{|l|}{ Age } & $82.84(6.58)$ & $72.96(6.30)$ & $t=7.06^{*}$ \\
\hline \multicolumn{2}{|l|}{ Years of education } & $\begin{array}{c}11.91^{\mathrm{a}}(3.82) \\
n(\%)^{\mathrm{b}}\end{array}$ & $\begin{array}{c}16.07(3.59) \\
n(\%)^{\mathrm{b}}\end{array}$ & $t=5.14 *$ \\
\hline \multirow[t]{2}{*}{ Gender } & Male & $17(50)$ & $21(38)$ & $\chi^{2}=0.83$ \\
\hline & Female & $17(50)$ & $34(63)$ & \\
\hline \multirow[t]{2}{*}{ Ethnicity } & White & $28(82)$ & $54(98)$ & $\chi^{2}=7.27$ \\
\hline & BME & $6(18)$ & $1(2)$ & \\
\hline \multirow[t]{5}{*}{ Marital status } & Single & $5(15)$ & $7(13)$ & $\chi^{2}=3.62$ \\
\hline & Married & $12(35)$ & $29(53)$ & \\
\hline & Separated & $0(0)$ & $1(2)$ & \\
\hline & Divorced & $6(18)$ & $7(13)$ & \\
\hline & Widowed & $11(32)$ & $11(20)$ & \\
\hline \multirow[t]{6}{*}{ Diagnosis $(\text { ICD } 10 \text { code) })^{c}$} & Alzheimer's disease — early onset (F00.0) & $1(3)$ & & \\
\hline & Alzheimer's disease—late onset (F00.1) & $20(59)$ & & \\
\hline & Alzheimer's disease—atypical/mixed type (F00.2) & $5(15)$ & & \\
\hline & Alzheimer's disease—unspecified type (F00.9) & $4(12)$ & & \\
\hline & Dementia in Parkinson's disease (F02.3) & $1(3)$ & & \\
\hline & Dementia-type unspecified (F0.3) & $3(9)$ & & \\
\hline
\end{tabular}

$* p<0.001$

${ }^{\mathrm{a}} N=33$

${ }^{\mathrm{b}}$ Percentages may not total 100 due to rounding

${ }^{\mathrm{c}}$ Dementia group only

function and overall cognition. If these correlations were present, it would be expected that performance on these measures predicted mindfulness performance over and above mood and premorbid IQ.
Table 2 Data for

neuropsychological and mood variables by group

\begin{tabular}{|c|c|c|c|c|}
\hline & \multicolumn{2}{|l|}{ Group } & & \\
\hline & $\operatorname{PWD}(N=34)$ & $\mathrm{OA}(N=55)$ & & \\
\hline Variable & $\begin{array}{l}\text { M } \\
(\mathrm{SD})\end{array}$ & $\begin{array}{l}\mathrm{M} \\
(\mathrm{SD})\end{array}$ & Test statistic & Effect size $(d)$ \\
\hline $\operatorname{ACE}(/ 100)$ & $\begin{array}{l}\text { Min-Max } \\
74^{\mathrm{a}} \\
(10.84)\end{array}$ & $\begin{array}{l}\text { Min-Max } \\
95.11 \\
(4.42)\end{array}$ & $t=10.67^{*}$ & 2.55 \\
\hline \multirow[t]{2}{*}{ Estimated IQ } & $\begin{array}{l}45-98 \\
101.92^{\text {a, b }}\end{array}$ & $\begin{array}{l}82-100 \\
118.70\end{array}$ & $t=6.16^{*}$ & 1.42 \\
\hline & $(14.00)$ & $(9.02)$ & & \\
\hline \multirow[t]{3}{*}{ TMT difference score } & $\begin{array}{l}75.90-126.40 \\
166.58^{\mathrm{a}}\end{array}$ & $\begin{array}{l}93.30-132.60 \\
42.98\end{array}$ & $t=8.80^{*}$ & 2.07 \\
\hline & $(76.36)$ & (35.94) & & \\
\hline & $38-274$ & $4-155$ & & \\
\hline \multirow[t]{3}{*}{ HADS (anxiety) (/21) } & 4.94 & 4.55 & $t=0.64$ & 0.14 \\
\hline & $(2.76)$ & $(2.89)$ & & \\
\hline & $1-12$ & $0-12$ & & \\
\hline \multirow[t]{3}{*}{ HADS (depression) $(/ 21)$} & 5.35 & 2.47 & $t=4.21^{*}$ & 0.95 \\
\hline & $(3.88)$ & $(1.82)$ & & \\
\hline & $0-14$ & $0-7$ & & \\
\hline
\end{tabular}

$* p=<0.001$

${ }^{\text {a }}$ Missing data $-N=33$

${ }^{\mathrm{b}}$ IQ estimates relate to premorbid IQ in PWD group 
Table 3 Results of Mann-Whitney $U$ tests with descriptive statistics for MBAS performance by group

\begin{tabular}{|c|c|c|c|c|c|c|c|c|c|c|}
\hline \multirow[b]{3}{*}{ Variable } & \multicolumn{6}{|l|}{ Group } & \multirow[b]{3}{*}{$d f$} & \multirow[b]{3}{*}{$U$} & \multirow[b]{3}{*}{$z$} & \multirow[b]{3}{*}{$r$} \\
\hline & \multicolumn{3}{|c|}{$\operatorname{PWD}(N=34)$} & \multicolumn{3}{|c|}{$\mathrm{OA}(N=55)$} & & & & \\
\hline & Median & Mode & Min-Max & Median & Mode & Min-Max & & & & \\
\hline \multirow[t]{2}{*}{ MBAS (with imputed data) } & 3 & 5 & $0-5$ & 4 & 4 & $0-5$ & 87 & $1036.50 *$ & 0.88 & 0.09 \\
\hline & \multicolumn{3}{|c|}{$\operatorname{PWD}(N=26)$} & \multicolumn{3}{|c|}{$\mathrm{OA}(N=55)$} & & & & \\
\hline MBAS (without imputed data) & 4 & 5 & $0-5$ & 4 & 4 & $0-5$ & 79 & $689.50^{* *}$ & -0.27 & 0.03 \\
\hline
\end{tabular}

$* p=0.38 ; * * p=0.79$

Where possible, further exploratory analyses were carried out for the dementia sample, to assess any associations between MBAS performance and the two putatively associated neurocognitive variables of overall cognition and cognitive flexibility (plus the potential nuisance variates of anxiety, depression and estimated premorbid IQ). Results reported include imputed MBAS data, though interpretations were unchanged when these data were excluded.

Spearman's rank order correlation was performed to assess the relationship between the MBAS and the TMT difference score. The correlation was statistically non-significant, with a medium effect size $\left(r_{s}(31)=0.323, p=0.067\right)$. The same analysis was performed to assess the relationship between MBAS and HADS (anxiety) scores, and again, the resulting correlation was statistically non-significant, with a small to medium effect size $\left(r_{s}(32)=0.181, p=0.306\right)$. Interpretations of effect sizes were based on Cohen's framework $(1988,1992)$, where $r=0.10$ represents a small effect size, $r=0.30$ a medium effect size and $r=0.50$ a large effect size. Correlational analyses for the MBAS task and the ACE, HADS (depression) and premorbid IQ were not performed, as assessment of bivariate scatterplots indicated the test's assumption of a monotonic relationship between variables was not met in these cases.

\section{Discussion}

This study investigated whether having a diagnosis of dementia was associated with reduced ability on a task of mindful attention. Our finding of no significant difference between a dementia group and members of an older adult comparison group on a task measuring the ability to mindfully attend to the breath suggests that diagnosis may not have a large effect. This was in spite of statistically significant differences between the groups on measures of overall cognition, estimated premorbid IQ, and executive ability, with people with dementia performing worse.

Further analysis aimed at exploring possible mechanisms influencing MBAS performance in people with dementia found a non-significant positive correlation between cognitive flexibility (the TMT difference score) and MBAS in the dementia sample. The medium effect size $(r=0.313)$ suggests that MBAS performance is also affected by or at least associated with other factors, and is insufficient strongly to support the construct validity of the MBAS task as previous papers have done (Frewen et al. 2011; Lai et al. 2015).

The expectation that people with dementia would perform significantly worse than the comparison group on the MBAS task was based in known and well-established differences (also reflected in the findings of the current study) between people with and without dementia, in areas which have a plausible theoretical link to the skills involved in optimal MBAS performance - cognition and cognitive flexibility. Given that the people with dementia group was a relatively high-functioning, community-dwelling sample, it is in fact possible that there was no underlying difference between the people with dementia and older adult group's ability to pay sustained, mindful attention to the breath. If this was the case, since some higher functioning people with dementia are able to engage in a task incorporating core elements of a key mindfulness meditation practice, mindfulness may be seen as a potential mechanism of change for future clinical interventions with this population. Although Churcher Clarke et al. (2017) did not find improvement on the MBAS task in those with dementia over the course of an intervention, it should be noted that the current study investigated a much higher functioning sample and it may be that in such a sample mindfulness ability is not affected in the same way. Further research would be needed to assess whether mindfulness abilities would improve in a similar sample over the course of a clinical intervention.

\section{Limitations and Future Research Directions}

Due to resource limitations, the first author was involved in administration of the test battery to the dementia group. She was not blinded to the status of these participants.

There was a period of time between initial diagnostic testing within the relevant memory services and subsequent identification and consenting of participants and their completion of the test battery; median (IQR) for the dementia sample were 291(525) days, respectively, and these lapses of time may 
have resulted in some participants' dementia further deteriorating since diagnosis.

We used an experience sampling measure with high face validity in this population and good evidence for validity in other populations. However, social desirability (in this case, the idea that paying attention was the 'correct' response) could have influenced performance (Paulhus 1991; Tourangeau and Yan 2007 (cited in Kaminska and Foulsham 2013)). Furthermore, valid self-report in the MBAS task is based on successfully meeting a number of cognitive demands. In particular, it requires set-shifting between the required focus of attention (the breath) and the instructions being provided, (short-term) retention of these instructions, accurate delayed recall of one's attentional state in the context of these instructions, and selection of the appropriate hand (left/right to indicate attention/distraction, depending on the condition). While delayed memory and other cognitive demands are reduced compared to a standard self-report task, they are by no means eliminated, and as can be seen from this brief summary, wider cognitive demands are fairly extensive.

Therefore, given that our samples differed significantly in cognitive abilities, these differences could have contributed to invalid responding in the dementia group. As detailed in the "Method" section, use of a simplified version of the MBAS task, adapted for a more severely impaired nursing home dementia sample (Churcher Clarke et al. 2017), was considered but rejected after piloting.

Although the MBAS task has ecological validity in measuring performance on a common mindfulness practice, the wider testing situation was less representative of a naturalistic meditation setting. Care is taken in formal meditation settings to aid focus and concentration by reducing or removing sources of external distraction. Such a level of control was difficult to achieve in participants' homes, where the majority of testing for the people with dementia sample took place. Sometimes family members/partners were present, occasionally presenting a distraction to the participant.

Finally, it is possible that there was a significant but small difference between groups on the MBAS which the study was underpowered to detect - scores between the groups did differ, with people with dementia having a lower median score. However, that difference was associated with a very small effect size of 0.09. Even if a higher-powered study had found this difference to be statistically significant, it is arguable that such a small effect size may not have clinical significance (Kazdin 1999; Shabbir and Sanders 2014).

Therefore, while the finding that there was no significant large effect size difference between those with dementia and those without on a measure of mindful attention may encourage further research on this area, interpretations of the study's outcomes must necessarily be tentative. The paucity of research in this area necessitated an initial proof of concept study, with exploratory use of a measure not previously validated for people with dementia. It is possible (as acknowledged above) that aspects of the methodology, of the measure itself, and the low power of the study affected the outcomes, and complicate their interpretation. Future research could usefully replicate the current study with a larger dementia sample, and focus in greater depth on exploring the elements of the measure and its administration which may have affected its validity, as a basis for developing a better validated measure. Studies could also consider alternative approaches to assessing mindful attention in a dementia sample which might further obviate the self-report element, for example, use of neuroimaging (Tang et al. 2015). If a validated measure can be established, research would then usefully incorporate a longitudinal element, to explore the clinically relevant area of whether this population can increase its level of mindful attention through practice.

Acknowledgments The authors would like to acknowledge the research participants who gave their time so generously and thank Noor Habib, Els Chadwick, and Janina Brede for their role in data collection.

Author Contributions CB and JS collaborated on the design and analysis of the study and the writing and editing of the final manuscript.

Funding This study was completed as part of a fellowship awarded to Joshua Stott by the Alzheimer's Society. Grant number 236 (AS-CTF-14005). This funding source had no involvement in the study design, collection, analysis, and interpretation of data, writing the manuscript, and in the decision to submit the manuscript for publication.

\section{Compliance with Ethical Standards}

Conflict of Interest The authors declare that they have no conflict of interest.

Research Involving Human Participants All procedures performed in the present study involving human participants were in accordance with the ethical standards of the institutional and/or national research committee and with the 1964 Helsinki Declaration and its later amendments or comparable ethical standards.

IRB approval for the study was provided by NRES Committee London-City Road \& Hampstead (REC Reference 14/LO/0554).

Informed Consent Informed consent was obtained from all individual participants included in the study.

Open Access This article is distributed under the terms of the Creative Commons Attribution 4.0 International License (http:// creativecommons.org/licenses/by/4.0/), which permits unrestricted use, distribution, and reproduction in any medium, provided you give appropriate credit to the original author(s) and the source, provide a link to the Creative Commons license, and indicate if changes were made.

\section{References}

Austin, M.-P. (2001). Cognitive deficits in depression: possible implications for functional neuropathology. The British Journal of Psychiatry, 178(3), 200-206. 
Bishop, S. R., Lau, M., Shapiro, S., Carlson, L., Anderson, N. D., Carmody, J., et al. (2006). Mindfulness: a proposed operational definition. Clinical Psychology: Science and Practice, 11(3), 230-241.

Bjelland, I., Dahl, A. A., Haug, T. T., \& Neckelmann, D. (2002). The validity of the Hospital Anxiety and Depression Scale: an updated literature review. Journal of Psychosomatic Research, 52(2), 69-77.

Bowie, C. R., \& Harvey, P. D. (2006). Administration and interpretation of the trail making test. Nature Protocols, 1(5), 2277-2281.

Chiesa, A., Calati, R., \& Serretti, A. (2011). Does mindfulness training improve cognitive abilities? A systematic review of neuropsychological findings. Clinical Psychology Review, 31(3), 449-464. https://doi.org/10.1016/j.cpr.2010.11.003.

Churcher Clarke, A., Chan, J. M. Y., Stott, J., Royan, L., \& Spector, A. (2017). An adapted mindfulness intervention for people with dementia in care homes: feasibility pilot study. International Journal of Geriatric Psychiatry(32), 12, e123-e131. https://doi.org/10.1002/ gps.4669.

Cohen, J. (1988). Statistical power analysis for the behavioral sciences (2nd ed.). Hillsdale, N.J: Lawrence Erlbaum.

Cohen, J. (1992). A power primer. Psychological Bulletin, 112(July), 155-159. https://doi.org/10.1037/0033-2909.112.1.155.

Dagnan, D., \& Mellor, K. (2009). Assessment of cognitive therapy skills for people with learning disabilities. Advances in Mental Health and Learning Disabilities, 3(4), 25-30. https://doi.org/10.1108/ 17530180200900036.

Derakshan, N., \& Eysenck, M. W. (2009). Anxiety, processing efficiency, and cognitive performance: new developments from attentional control theory. European Psychologist, 14(2), 168-176.

Enache, D., Winblad, B., \& Aarsland, D. (2011). Depression in dementia: epidemiology, mechanisms, and treatment. Current Opinion in Psychiatry, 24(6), 461-472.

Frewen, P., Unholzer, F., Logie-Hagan, K. R. J., \& MacKinley, J. D. (2014). Meditation breath attention scores (MBAS): test-retest reliability and sensitivity to repeated practice. Mindfulness, 5(2), 161169

Frewen, P., Lundberg, E., MacKinley, J., \& Wrath, A. (2011). Assessment of response to mindfulness meditation: meditation breath attention scores in association with subjective measures of state and trait mindfulness and difficulty letting go of depressive cognition. Mindfulness, 2(4), 254-269.

Frewen, P., Evans, E. M., Maraj, N., Dozois, D. J. A., \& Partridge, K. (2008). Letting go: mindfulness and negative automatic thinking. Cognitive Therapy and Research, 32(6), 758-774.

Gelman, A., \& Hill, J. (2006). Data analysis using regression and multilevel/hierarchical models. Cambridge: Cambridge University Press.

González-Salvador, M. T., Arango, C., Lyketsos, C. G., \& Barba, A. C. (1999). The stress and psychological morbidity of the Alzheimer patient caregiver. International Journal of Geriatric Psychiatry, 14(9), 701-710.

Graham, J. W. (2009). Missing data analysis: making it work in the real world. Annual Review of Psychology, 60, 549-576 Retrieved from http://www.stats.ox.ac.uk/ snijders/SM_MissingData.pdf.

Hebblethwaite, A., Jahoda, A., \& Dagnan, D. (2011). Talking about reallife events: an investigation into the ability of people with intellectual disabilities to make links between their beliefs and emotions within dialogue. Journal of Applied Research in Intellectual Disabilities, 24(6), 543-553.

Herrero, M. J., Blanch, J., Peri, J. M., De Pablo, J., Pintor, L., \& Bulbena, A. (2003). A validation study of the hospital anxiety and depression scale (HADS) in a Spanish population. General Hospital Psychiatry, 25(4), 277-283.

Heun, R., Papassotiropoulos, A., \& Jennssen, F. (1998). The validity of psychometric instruments. Psychiatry, 13(6), 368-380.

Higgins, J. P. T., \& Green, S. (eds). Cochrane handbook for systematic reviews of interventions. Version 5.1.0 (updated March 2011). The
Cochrane Collaboration, 2011. Retrieved from https://handbook. cochrane.org

Holdnack, J. A., Schoenberg, M. R., Lange, R. T., \& Iverson, G. L. (2013). Predicting premorbid ability for WAIS-IV, WMS-IV and WASI-II. In Holdnack, J. A.. Drozdick, L. W., Weiss, L. G., \& Iverson, G. L. (Eds.), Practical resources for the mental health professional. WAISIV, WMS-IV, and ACS: Advanced clinical interpretation (217-278). San Diego, CA, US: Elsevier Academic Press.

Hsieh, S., Schubert, S., Hoon, C., Mioshi, E., \& Hodges, J. R. (2013). Validation of the Addenbrooke's Cognitive Examination III in frontotemporal dementia and Alzheimer's disease. Dementia and Geriatric Cognitive Disorders, 36(3-4), 242-250.

Johnston, M., Pollard, B., \& Hennessey, P. (2000). Construct validation of the Hospital Anxiety and Depression Scale with clinical populations. Journal of Psychosomatic Research, 48(6), 579-584.

Joyce, T., Globe, A., \& Moody, C. (2006). Assessment of the component skills for cognitive therapy in adults with intellectual disability. Journal of Applied Research in Intellectual Disabilities, 19(1), 17-23.

Kales, H. C., Chen, P., Blow, F. C., Welsh, D. E., \& Mellow, A. M. (2005). Rates of clinical depression diagnosis, functional impairment, and nursing home placement in coexisting dementia and depression. The American Journal of Geriatric Psychiatry, 13(6), 441449.

Kaminska, O. \& Foulsham, T. (2013). Understanding sources of social desirability bias in different modes: evidence from eye-tracking. ISER Working Paper Series, No. 2013-04. Colchester: ISER.

Kazdin, A. E. (1999). The meanings and measurement of clinical significance. Journal of Consulting and Clinical Psychology, 67(3), 332339. https://doi.org/10.1037/0022-006X.67.3.332.

Kiel, C. (2007). G*Power 3: a flexible statistical power analysis program for the social, behavioral, and biomedical sciences. Behavioural Research Methods, 39(2), 175-191.

Kortte, K. B., Horner, M. D., \& Windham, W. K. (2002). The trail making test, part B: cognitive flexibility or ability to maintain set? Applied Neuropsychology, 9(2), 106-109.

Kunik, M. E., Snow, A. L., Molinari, V. A., Menke, T. J., Souchek, J., Sullivan, G., \& Ashton, C. M. (2003). Health care utilization in dementia patients with psychiatric comorbidity. The Gerontologist, 43(1), 86-91.

Lai, C., MacNeil, B., \& Frewen, P. (2015). A comparison of the attentional effects of single-session mindfulness meditation and Fp-HEG neurofeedback in novices. Mindfulness, 6(5), 1012-1020.

Law, E., Connelly, P. J., Randall, E., McNeill, C., Fox, H. C., Parra, M. A., et al. (2013). Does the Addenbrooke's Cognitive Examinationrevised add to the Mini-Mental State Examination in established Alzheimer disease? Results from a national dementia research register. International Journal of Geriatric Psychiatry, 28(4), 351-355. https://doi.org/10.1002/gps.3828.

Lezak, M. D., Howieson, D. B., Bigler, E. D., \& Tranel, D. (2012). Neuropsychological assessment. Oxford: Oxford University Press.

Liu, X., Wei, X., Wang, Y., Williams, J. M. G., Geng, Y., Zhang, Q., \& Liu, X. (2013). Can inner peace be improved by mindfulness training: a randomized controlled trial. Stress and Health, 31(3), 245254.

mindfulnessteachersuk.org.uk (November 2011). UK network for mindfulness-based teachers: good practice guidelines for teaching mindfulness-based courses. Retrieved from http:// mindfulnessteachersuk.org.uk.

Moore, A., \& Malinowski, P. (2009). Meditation, mindfulness and cognitive flexibility. Consciousness and Cognition, 18(1), 176-186.

Orgeta, V., Qazi, A., Spector, A., \& Orrell, M. (2015). Psychological treatments for depression and anxiety in dementia and mild cognitive impairment: systematic review and meta-analysis. The British Journal of Psychiatry, 207(4), 293-298. 
Paulhus, D. (1991). Measurement and control of response bias. In Measures of personality and social psychological attitudes (pp. 17-59). San Diego: Academic Press.

Piet, J., \& Hougaard, E. (2011). The effect of mindfulness-based cognitive therapy for prevention of relapse in recurrent major depressive disorder: A systematic review and meta-analysis. Clinical Psychology Review., 31(6), 1032-1040. https://doi.org/10.1016/j. cpr.2011.05.002.

Prince, M., Knapp, M., Guerchet, M., McCrone, P., Prina, M., ComasHerrera, A., Wittenberg, R., Adelaja, B., Hu, B., King, D., Rehill, A., \& Salimkumar, D. (2014). Dementia UK: Update. Retrieved from https://www.alzheimers.org.uk

Prince, M., Wimo, A., Guerchet, M., Gemma-Claire, A., Wu, Y.-T., \& Prina, M. (2015). World Alzheimer report 2015: The global impact of dementia - an analysis of prevalence, incidence, cost and trends. Retrieved from https://www.alz.co.uk

Rapp, M. A., Schnaider-Beeri, M., Wysocki, M., Guerrero-Berroa, E., Grossman, H. T., Heinz, A., \& Haroutunian, V. (2011). Cognitive decline in patients with dementia as a function of depression. American Journal of Geriatric Psychiatry, 19(4), 357-363.

Samaras, N., Herrmann, F. R., Samaras, D., Lang, P. O., Canuto, A., Forster, A., et al. (2013). The Hospital Anxiety and Depression Scale: low sensitivity for depression screening in demented and non-demented hospitalized elderly. International Psychogeriatrics, 25(1), 82-87.

Sanchez-Cubillo, I., Perianez, J. A., Adrover-Roig, D., RodriguezSanchez, J. M., Rios-Lago, M., Tirapu, J., \& Barcelo, F. (2009). Construct validity of the Trail Making Test: role of task-switching, working memory, inhibition/interference control, and visuomotor abilities. Journal of the International Neuropsychological Society, $15,438-450$.

Napa Scollon, C., Prieto, C. K., \& Diener, E. (2009). Experience sampling: promises and pitfalls, strength and weaknesses. In E. Diener (Ed.), Assessing well-being. Social Indicators Research Series, 39. Dordrecht: Springer.

Shabbir, S. H., \& Sanders, A. E. (2014). Clinical significance in dementia research: a review of the literature. American Journal of Alzheimer's Disease and Other Dementias, 29(6), 492-497. https://doi.org/10. $1177 / 1533317514522539$.

Shin, I.-S., Carter, M., Masterman, D., Fairbanks, L., \& Cummings, J. L. (2005). Neuropsychiatric symptoms and quality of life in Alzheimer disease. American Journal of Geriatric Psychiatry, 13(6), 469-474.

Snaith, R. P., \& Zigmond, A. S. (1983). The Hospital Anxiety and Depression Scale. Acta Psychiatrica Scandinavica, 67, 361-370.

Spector, A., Thorgrimsen, L., Woods, B., Royan, L., Davies, S., Butterworth, M., \& Orrell, M. (2003). Efficacy of an evidence- based cognitive stimulation therapy programme for people with dementia: randomised controlled trial. British Journal of Psychiatry, 183(Sept), 248-254. https://doi.org/10.1192/bjp.183.3.248.

Stott, J., Scior, K., Mandy, W., \& Charlesworth, G. (2017a). Dementia screening accuracy is robust to premorbid IQ variation: evidence from the Addenbrooke's Cognitive Examination-III and the Test of Premorbid Function. Journal of Alzheimer's Disease, 57(4), 12931302.

Stott, J., Spector, A., Orrell, M., Scior, K., Sweeney, J., \& Charlesworth, G. (2017b). Limited validity of the Hospital Anxiety and Depression Scale (HADS) in dementia: evidence from a confirmatory factor analysis. International Journal of Geriatric Psychiatry, 32(7), 805-813. https://doi.org/10.1002/gps.4530.

Strauss, C., Cavanagh, K., Oliver, A., \& Pettman, D. (2014). Mindfulness-based interventions for people diagnosed with a current episode of an anxiety or depressive disorder: a meta-analysis of randomised controlled trials. PLoS One, 9(4) Retrieved from http:// www.ncbi.nlm.nih.gov.

Suh, G., \& Yeon, B. K. (2005). Mortality in Alzheimer's disease: a comparative prospective Korean study in the community and nursing homes. International Journal of Geriatric Psychiatry, 20(1), 26-34.

Tang, Y.-Y., Hölzel, B. K., \& Posner, M. I. (2015). The neuroscience of mindfulness meditation. Nature Reviews Neuroscience, 16(5), 312312. https://doi.org/10.1038/nrn3954.

Tourangeau, R., \& Yan, T. (2007). Sensitive questions in surveys. Psychological Bulletin, 133(5), 859-883. https://doi.org/10.1037/ 0033-2909.133.5.859.

Velayudhan, L., Ryu, S.-H., Raczek, M., Philpot, M., Lindesay, J., Critchfield, M., \& Livingston, G. (2014). Review of brief cognitive tests for patients with suspected dementia. International Psychogeriatrics, 26(8), 1247-1262.

Wechsler, D. (2011). Test of premorbid functioning - UK version (TOPF UK). London: Pearson.

Winblad, B., Palmer, K., Kivipelto, M., Jelic, V., Fratiglioni, L., Wahlund, L. O., ... Petersen, R. C. (2004). Mild cognitive impairment Beyond controversies, towards a consensus: Report of the International Working Group on Mild Cognitive Impairment. Journal of Internal Medicine, 256(3), 240-246. https://doi.org/10. $1111 / \mathrm{j} .1365-2796.2004 .01380 . x$.

World Health Organization. (2016). International statistical classification of diseases and related health problems (10th revision). Geneva: World Health Organization.

World Medical Association. (2013). World medical association declaration of Helsinki ethical principles for medical research involving human subjects. JAMA, 310(20), 2191-2194. https://doi.org/10. 1001/jama.2013.281053. 